\title{
A Comparison of Mycophenolate Mofetil with Ciclosporine for the Treatment of Chronic Plaque-Type Psoriasis
}

\author{
Stefan Beissert ${ }^{\mathrm{a}, \mathrm{e}} \quad$ Sylvia Pauser ${ }^{\mathrm{a}} \quad$ Michael Sticherling $^{\mathrm{b}, \mathrm{c}} \quad$ Uta Frieling $^{\mathrm{a}}$ \\ Klaus-Dieter Loske ${ }^{a}$ Peter J. Frosch ${ }^{d}$ Ingo Haase ${ }^{e}$ Thomas A. Luger ${ }^{\text {a }}$ \\ Departments of Dermatology, a University of Münster, Münster, ${ }^{b}$ University of Leipzig, Leipzig, ' University of \\ Erlangen, Erlangen, ${ }^{d}$ Klinikum Dortmund $\mathrm{GmbH}$, Dortmund, and e University of Cologne, Cologne, Germany
}

\section{Key Words}

Psoriasis, clinical study $\cdot$ Immunosuppression $\cdot$ T cells

\begin{abstract}
Aims: To compare the efficacy of ciclosporine (CsA) versus mycophenolate mofetil (MMF) in psoriasis, a randomized trial was conducted. Methods: A prospective multicenter randomized open-label clinical trial was performed to compare two parallel groups of patients with chronic plaque psoriasis undergoing different treatments. Therefore, a total of $54 \mathrm{pa}-$ tients with psoriasis were randomly assigned to treatment with either CsA ( $2.5 \mathrm{mg} / \mathrm{kg}$ body weight) or MMF ( $2 \mathrm{~g}$ daily) for 12 weeks, and the drug doses were adjusted according to response. The psoriasis area and severity index (PASI) was used to assess the clinical severity of psoriasis. The primary outcome of this trial was the time to disease relapse. Safety, PASI scores and psoriasis disability index (PDI) were assessed as secondary outcome. Results: There was no difference in time to disease relapse between the two groups. After 12 weeks of treatment, the mean PASI score $( \pm S D)$ decreased from $24.6 \pm 11.1$ to $6.6 \pm 7.3$ in the CsA group $(n=27)$ and from $22.4 \pm 9.2$ to $10.6 \pm 6.7$ in the MMF group $(n=27 ; p=$ 0.02). The side effects, time to remission and PDI were similar in both groups. Conclusions: After 12 weeks, CsA demonstrated a significantly superior efficacy in psoriasis compared to MMF.

Copyright $\odot 2009$ S. Karger AG, Basel
\end{abstract}

\section{Introduction}

Chronic plaque psoriasis is an immune-mediated inflammatory skin disease with an estimated worldwide prevalence of $2 \%$ in the Caucasian population [1-4]. Especially patients with severe psoriasis have an increased mortality rate pointing to the significant unmet need which remains for safe and effective treatment [5]. The current therapeutic strategy for the treatment of moderate to severe psoriasis is based on multiple alterations in treatment regimens to decrease the cumulative risks of adverse events $[6,7]$. The actual choice of therapy is guided by considerations such as the severity of disease, effectiveness of medication, potential side effects, the patient's quality of life and the convenience of treatment [4, $6,8,9]$.

In the pathogenesis of psoriasis, aberrant activation of leukocytes including $\mathrm{T}$ cells appears to play an important role, and this finding has suggested that intracellular signaling pathways induced upon $\mathrm{T}$ cell receptor stimulation may represent appropriate therapeutic targets $[8,9]$. Hence, ciclosporine (CsA), which interferes with the calcineurin-catalyzed movement of the nuclear factor of ac-

Stefan Beissert has served as consultant and paid speaker for Roche. Thomas A. Luger has served as consultant for Roche.

\section{KARGER}

Fax +41613061234

E-Mail karger@karger.ch

www.karger.com
(C) 2009 S. Karger AG, Basel

$1018-8665 / 09 / 2192-0126 \$ 26.00 / 0$

Accessible online at:

www.karger.com/drm
Stefan Beissert, MD

Professor of Dermatology

University of Münster, Von-Esmarch-Strasse 58

DE-48149 Münster (Germany)

Tel. +492518356565, Fax +49251835 7479, E-Mail beisser@uni-muenster.de 
Fig. 1. Trial profile.

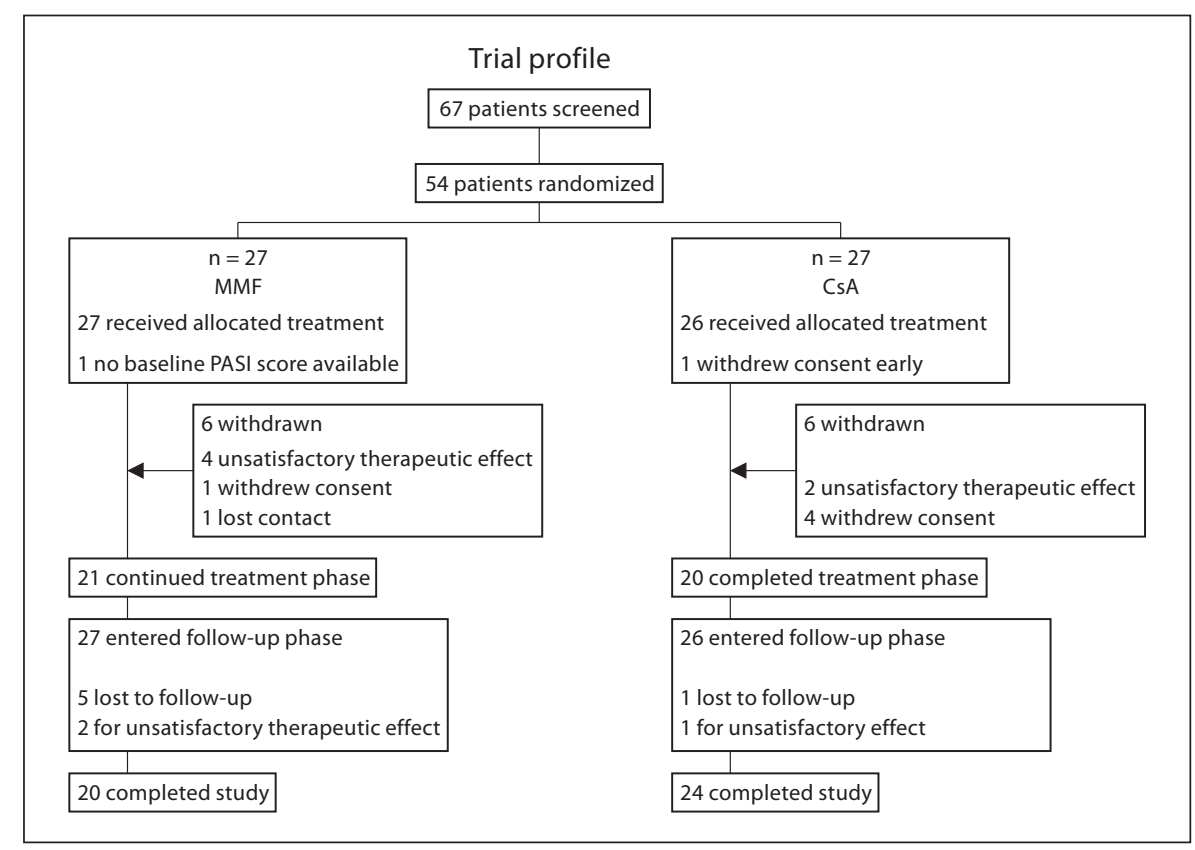

tivated T lymphocytes into the nucleus to induce cytokine genes required for $\mathrm{T}$ cell activation such as interleukin 2, has been successfully introduced for the treatment of psoriasis [10-16].

Besides the therapeutic blockade of calcineurin-mediated $\mathrm{T}$ cell activation, inhibition of DNA replication in lymphocytes was shown to control psoriasis. In this context, mycophenolic acid, which inhibits inositol monophosphate dehydrogenase (IMPDH), a key enzymatic pathway during lymphocyte activation, has been successfully used in the therapy of psoriasis for more than three decades [17-19]. More recently, mycophenolate mofetil (MMF) was shown to have a beneficial therapeutic effect in psoriasis $[20,21]$. To determine the therapeutic effects of blocking calcineurin signaling or IMPDH activity for the control of psoriasis, we conducted a randomized comparison of CsA with MMF as monotherapy for moderateto-severe chronic plaque psoriasis. In this trial, the clinical effectiveness, side effects and quality of life were evaluated.

\section{Methods}

Patients

Patients were recruited from 5 academic departments of dermatology in Germany. The first patient consented to participate on September 11,2000, and the last patient visit occurred on August 28,2006 . After providing written informed consent, men and women (aged $\geq 18$ and $\leq 75$ years) were eligible if they had the diagnosis of plaque psoriasis, had not previously been treated with CsA or MMF, were candidates for phototherapy or systemic treatment, and had a baseline score on the psoriasis area and severity index (PASI) of $\geq 10$ (on a scale of $0-72$, with higher scores indicating severer disease).

Patients were ineligible if they had nonplaque psoriasis, had recent serious systemic or local infections, had active or latent tuberculosis, had a known malignancy, had uncontrolled hypertension, had severe cardiovascular, pulmonary, cerebral, neurological or hematological disease, had received biological or investigational drugs within the last 4 weeks, or had received conventional systemic psoriasis treatment or phototherapy within the previous 2 weeks. Patients were also excluded if they were pregnant, breast-feeding or noncompliant with an effective regimen of contraception.

Patients who were eligible underwent the following laboratory tests prior to randomization and at weeks $2,4,6,8,10$ and 12: complete blood count, assays of electrolytes, serum creatinine, blood urea nitrogen, aspartate aminotransferase, alanine aminotransferase, alkaline phosphatase, $\gamma$-glutamyltransferase, lactate dehydrogenase and bilirubin. If a relevant abnormality (defined as $25 \%$ out of the normal range) in any of the laboratory values was noted during treatment, the test was repeated at each visit until the results returned to normal. This trial was approved by the ethics committee of each center.

\section{Study Design}

This randomized open-label trial was designed as a superiority study achieving $80 \%$ power to significantly detect an improvement in the relapse-free rate at the end of the observation period from 40 to $60 \%$ by the experimental treatment using Nsurv software (idv, Gauting, Germany). The randomization was performed 
Fig. 2. Rate without relapse of psoriasis in all evaluable patients.

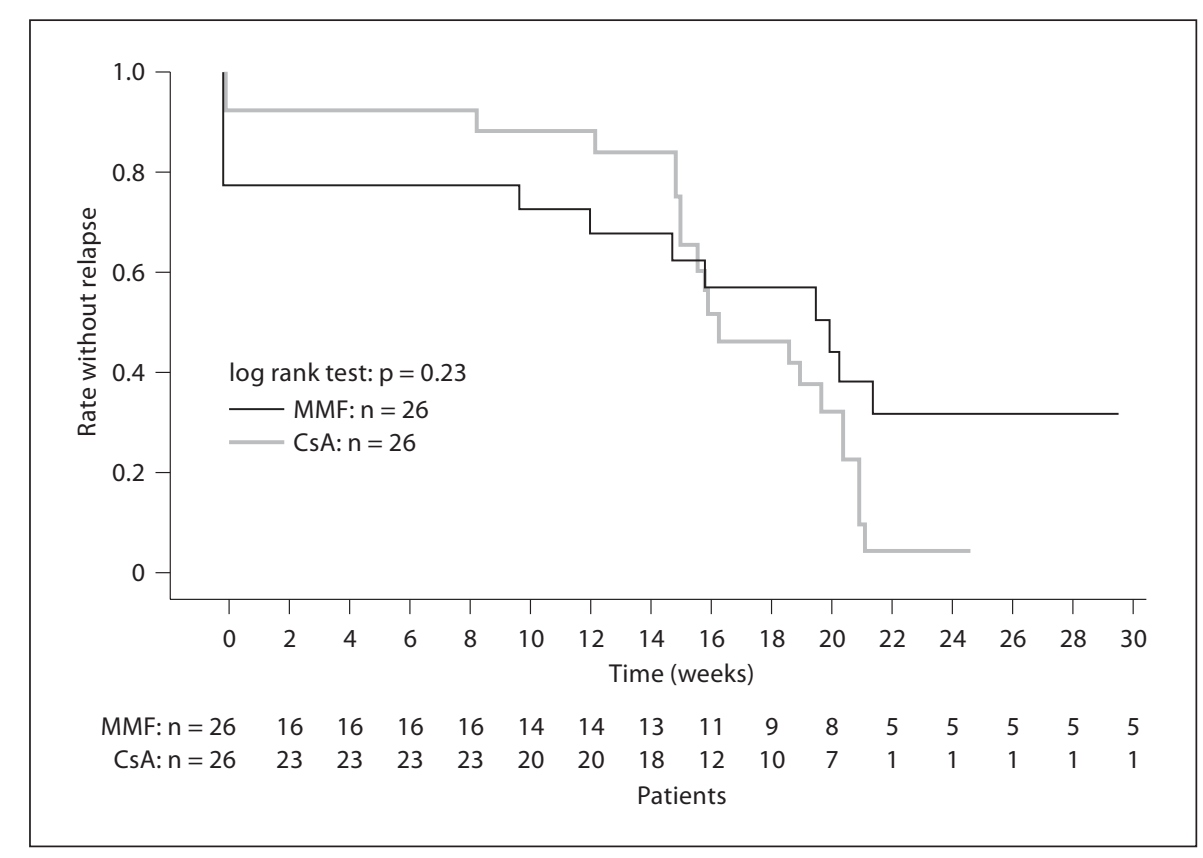

centrally by fax using computer-generated randomization lists consisting of permutated blocks with varying size. Eligible patients who had given written informed consent were randomly assigned to receive 12 weeks of treatment with either CsA or MMF (fig. 1). In one treatment arm, patients were allocated to receive CsA (Sandimmun ${ }^{\circledR}$ Optoral, Novartis Pharma, Nürnberg, Germany) at a dose of $1.25 \mathrm{mg} / \mathrm{kg}$ body weight (BW) given twice daily. In the other arm, patients received MMF (Cellcept ${ }^{\circledR}$, Roche Pharma AG, Grenzach-Wyhlen, Germany) at a dose of $1 \mathrm{~g}$ given twice daily. At week 6, patients with a PASI improvement of $>60 \%$ received a reduced CsA dose of $1.25 \mathrm{mg} / \mathrm{kg} \mathrm{BW}$ given once per day or a reduced MMF dose of $500 \mathrm{mg}$ given twice daily. In patients with a PASI improvement of $\leq 25 \%$, CsA medication was increased from week 7 to 12 to $2.5 \mathrm{mg} / \mathrm{kg}$ BW twice daily or the MMF dose was increased to $1.5 \mathrm{~g}$ twice daily. After 12 weeks of treatment, patients were monitored for another 12 weeks. They returned for evaluation every 3 weeks. During the follow-up period, topical treatment for psoriasis was allowed, if necessary, with emollients such as water-in-oil or oil-in-water emulsions reflecting normal daily practice. Drugs known to interfere with psoriasis or with the systemic treatments were not allowed. Study medication was counted at each visit until week 12 to assess patient compliance.

\section{Efficacy and Safety Evaluations}

Efficacy evaluations were performed at weeks 1, 2, 4, 6, 8, 10, $12,15,18,21$ and 24. The PASI combines an assessment of the extent of body surface involvement in 4 anatomical regions (head, trunk, upper and lower extremities) and the severity of desquamation, erythema and plaque thickness in each region, yielding an overall score of 0 (for no psoriasis) to 72 (for severest psoriasis) [22]. A minimal response was defined as a $\geq 25 \%$ improvement of the baseline PASI score. The PASI score was determined by trained assessors who were unaware of the treatment assignments. The
German version of the psoriasis disability index (PDI) was completed by the patients and measures whether psoriasis has an effect on the patient's quality of life (scores ranging from $0=$ no effect at all on patient's life to $45=$ extremely large effect on patient's life) $[23,24]$. The safety as well as tolerability of CsA or MMF was assessed by passively collecting adverse events and routine laboratory parameters up to week 24 . Severity was graded according to the National Cancer Center Institute common toxicity criteria.

\section{Statistical Analysis}

Baseline demographics and clinical characteristics were summarized descriptively. Main efficacy analyses were performed in the intention-to-treat population, which included all randomized patients. Missing values at week 12 or during the follow-up were replaced with the most recently available values for efficacy and safety; missing data at other time points were not imputed. Time to disease relapse, defined as a $50 \%$ increase in the PASI score in relation to the lowest intermittently achieved score (or baseline, if no response was observed at all), as the primary endpoint of the study was estimated according to the method of Kaplan and Meier and compared using the log rank test $[25,26]$. In the KaplanMeier graph (fig. 2), patient data were censored on the last documented observation point. Missing values at week 12 or during follow-up were replaced with the most recently available values e.g. for efficacy or safety, missing data at other time points were not imputed. All other analyses presented are of descriptive or hypothesis-generating nature. Dichotomous and ordered categorical data, such as response rate and PASI score improvement categories, were analyzed with Fisher's exact or a corresponding exact permutation version of the Cochrane-Armitage trend test, respectively. With respect to the PASI, PDI scores and day counts, the Wilcoxon test was applied for between-group comparisons. All reported p values are two-sided. 


\section{Results}

\section{Characteristics of Patients}

Of the 52 patients randomly assigned to the two treatment arms, 37 were men (table 1). Baseline demographics and disease characteristics were similar among the trial groups (table 1). Most patients (>90\%) in both groups had been treated for psoriasis with phototherapy and/or fumaric acid esters before. Of the 27 patients allocated to receive CsA, 1 withdrew informed consent early. The mean average PASI score ranged from $22.4 \pm 9.2$ (MMF group) to $24.6 \pm 11.1$ (CsA group); 49/52 of patients had been treated for psoriasis before. The mean average duration of psoriasis was $15.2 \pm 13.1$ years in the MMF group and $16.1 \pm 14.2$ years in the CsA group.

\section{Efficacy}

The rate of response was high in the CsA-treated group since $24 / 26$ of the patients in this group had reached the threshold for a minimal response after 12 weeks of treatment before the medication was discontinued. In the MMF-treated group, the rate of response was lower since in $20 / 27$ of the patients in this group the threshold for minimal response was documented (difference of means: $15 \%, 95 \%$ confidence interval: $6 \%$ in favor of MMF to $37 \%$ in favor of CsA; $\mathrm{p}=0.25$ ). The data in table 2 show the mean PASI scores during the study period. Twelve weeks after randomization, the mean PASI score $( \pm S D)$ was 6.6 \pm 7.3 in the CsA group and $10.6 \pm 6.7$ in the MMF group of patients. The relative reduction in PASI scores from baseline to 12 weeks of treatment was $79 \%$ in the CsA group compared to $49 \%$ in the MMF group of patients (difference of means: 25\%, 95\% confidence interval: 9$40 \%, \mathrm{p}=0.004)$.

Eight of 26 patients in the CsA group compared to 1/27 in the MMF group had an almost complete remission (defined as a reduction of the baseline PASI by $\geq 90 \%$ ) during the 12 weeks of treatment $(p=0.024)$. Partial remission (defined as a reduction of the baseline PASI by $\geq 75 \%$ ) was achieved in $8 / 26$ patients of the CsA group and in 6/27 patients of the MMF group. The time needed to reach a complete and/or a partial remission was 26.6 \pm 15.5 days in the CsA group and $28.3 \pm 14.5$ days in the MMF group $(\mathrm{p}=0.87)$.

After 6 weeks of treatment, an improvement in PASI scores in the CsA group was achieved as follows: PASI $\geq 90 \%$ in $2 / 24$ patients, PASI $\geq 75 \%$ in $8 / 24$ patients, and PASI $\geq 50 \%$ in $10 / 24$ patients. Accordingly, the CsA dose was reduced in $10 / 24$ patients, maintained in $10 / 24$ patients and increased in 4/24 patients. In the MMF group
Table 1. Baseline characteristics of patients

\begin{tabular}{lcc}
\hline & MMF & CsA \\
\hline Males & $17(65)$ & $20(77)$ \\
Females & $9(35)$ & $6(23)$ \\
Age, years & $40 \pm 13.2$ & $42.9 \pm(14.1)$ \\
Duration of psoriasis, years & $15.2 \pm 13.1$ & $16.1 \pm 14.2$ \\
Patients treated previously & & \\
$\quad$ Yes & $25(96)$ & $24(92)$ \\
$\quad$ No & $1(4)$ & $2(8)$ \\
PASI score & $22.4 \pm 9.2$ & $24.6 \pm 11.1$ \\
Blood pressure, mm Hg & & \\
$\quad$ Systolic & $129 \pm 15.1$ & $130.6 \pm 12.9$ \\
$\quad$ Diastolic & $81.3 \pm 7.9$ & $82.3 \pm 7.9$ \\
Body weight, kg & $85 \pm 19.4$ & $86.7 \pm 12.6$ \\
\hline
\end{tabular}

Data are means $\pm \mathrm{SD}$ or numbers with percentages in parentheses.

Table 2. Clinical response at week 12

\begin{tabular}{lcc}
\hline & MMF & CsA \\
\hline PASI & & \\
Number & 16 & 21 \\
Mean \pm SD & $10.6 \pm 6.7$ & $6.6 \pm 7.3$ \\
Median & 8.2 & 3.6 \\
Interquartile range & $5.9-13.9$ & $2-9.4$ \\
p value (Wilcoxon test) & \multicolumn{2}{l}{} \\
Improvement in PASI & \multicolumn{2}{l}{} \\
At least 50\% & $5(31 \%)$ & $5(24 \%)$ \\
At least 75\% & $2(12 \%)$ & $6(29 \%)$ \\
At least 90\% & $1(6 \%)$ & $6(29 \%)$ \\
p value (Wilcoxon test) & \multicolumn{2}{l}{0.01} \\
\hline
\end{tabular}

after 6 weeks of treatment, an improvement in PASI scores was achieved as follows: PASI $\geq 90 \%$ in $0 / 23$ patients, PASI $\geq 75 \%$ in $0 / 23$ patients and PASI $\geq 50 \%$ in $15 / 23$ patients. In the MMF group, the study medication was reduced after 6 weeks in $0 / 23$ patients, maintained in $15 / 23$ patients and increased in $8 / 23$ patients.

No significant differences between the groups were found in the duration of either partial remission or complete remission after oral treatment had been stopped. The rate of recurrence of psoriasis (defined as an increase in the lowest documented PASI score in that patient by $\geq 50 \%$ ) in all patients in the CsA group compared to all patients in the MMF group was similar (fig. 2). 
Table 3. Adverse events

\begin{tabular}{lllllll}
\hline & \multicolumn{2}{l}{$M$} & & \multicolumn{2}{l}{ CsA $(\mathrm{n}=26)$} \\
\cline { 2 - 3 } \cline { 6 - 7 } & grade 3 & grade 4 & & grade 3 & grade 4 \\
\hline Bilirubin & $3(11)$ & - & & $3(12)$ & - \\
ALT/AST & $1(4)$ & - & & - & - \\
GGT/LDH & $2(7)$ & - & & - & - \\
Uric acid & - & $1(4)$ & & - & $1(4)$ \\
Hypertension & - & - & & $2(8)$ & - \\
Hypokalemia & - & - & & - & $1(4)$ \\
\hline
\end{tabular}

ALT $=$ Alanine aminotransferase; AST $=$ aspartate aminotransferase; GGT $=\gamma$-glutamyltransferase; $\mathrm{LDH}=$ lactate dehydrogenase. Data are numbers with percentages in parentheses.

Table 4. PDI score

\begin{tabular}{|c|c|c|}
\hline & MMF & CsA \\
\hline \multicolumn{3}{|l|}{ At randomization } \\
\hline Number & 21 & 23 \\
\hline Mean $\pm \mathrm{SD}$ & $35.4 \pm 19$ & $39.2 \pm 14.1$ \\
\hline Median & 35.6 & 40 \\
\hline $\mathrm{p}$ value (Wilcoxon test) & \multicolumn{2}{|c|}{0.38} \\
\hline \multicolumn{3}{|l|}{ Week 12} \\
\hline Number & 9 & 15 \\
\hline Mean $\pm \mathrm{SD}$ & $21 \pm 16.9$ & $16.7 \pm 14.5$ \\
\hline Median & 17.8 & 13.3 \\
\hline $\mathrm{p}$ value (Wilcoxon test) & \multicolumn{2}{|c|}{0.76} \\
\hline
\end{tabular}

\section{Safety}

Overall, 14 severe (grade 3 ) or life-threatening (grade 4) side effects were reported in 53 patients (table 3 ). In $7 / 27$ patients randomized to the MMF group, grade 3 or 4 side effects were documented compared to $7 / 26$ patients from the CsA group. The results in table 3 demonstrate that the side effects, which are typically associated with the immunosuppressant drugs used in this study, were similar between treatment arms. However, 2 psoriasis patients (out of 26) randomized to the CsA group developed grade 3 hypertension compared to no patients (out of 27) in the MMF group $(\mathrm{p}=0.11)$. To investigate the liver function, serum concentrations of aspartate aminotransferase, alanine aminotransferase, lactate dehydrogenase as well as $\gamma$-glutamyltransferase were determined and classified according to toxicity grades. Three out of 27 psoriasis patients in the MMF group showed grade 3 elevated aspartate/alanine aminotransferase or $\gamma$-glutamyltransferase/lactate dehydrogenase serum concentrations compared to $0 / 26$ patients in the CsA group $(p=0.24)$. One patient in the CsA group received oral antibiotic treatment for positive Lyme serological testing after an arthropod bite.

\section{Quality of Life}

At randomization no marked differences were found in the PDI values between the two treatment groups (table 4). Both immunosuppressant medications induced improvement of life quality within each treatment group.

\section{Discussion}

In this randomized open-label trial, the therapeutic efficacies of interference with calcineurin signaling or inhibition of IMPDH activity have been compared for the systemic treatment of patients with moderate-to-severe chronic plaque psoriasis. This trial was originally designed as a superiority study, which had required a higher patient number. Due to slow and further decreasing accrual, the trial had to be stopped prematurely and the obtained data were subsequently analyzed. The major reason for this was that during the recruitment phase of this trial many competing clinical investigations were launched to scrutinize the efficacy of several monoclonal antibodies such as adalimumab, ustekinumab or godalimumab for the treatment of psoriasis and many patients asked to participate in those studies to obtain treatment with 'biologicals'. As a consequence, the power is inadequate for a phase III trial, and all analyses should be rather regarded as explorative. Nevertheless, the presented results suggest that CsA was significantly more effective in decreasing the PASI score in week 12 after treatment had been started. It should be noted that since CsA treatment induced a stronger and faster reduction in PASI scores these patients had more room to relapse. Since relapse rates were an end point of this investigation, the trial design might bias the results in favor of a less effective treatment. The follow-up period of 12 weeks after discontinuation of treatment with either medication might be too short to completely and absolutely determine the relapse rates of disease as a primary end point of this study in both treatment arms. In a number of psoriasis trials, different follow-up periods, e.g. 12-16 weeks, have been reported [27]. Hence, there is no standard follow-up period for clinical investigations in psoriasis. Per- 
haps the elimination of the study medication in patients under detectable levels should guide future study designs in psoriasis since e.g. injected antibodies are detectable in the patient's serum weeks after discontinuation of treatment. The data presented in this investigation show in figure 2 that after 24 weeks ( 12 weeks treatment, 12 weeks follow-up) the vast majority of patients had experienced a recurrence of psoriasis. The findings may suggest that blocking calcineurin signaling in psoriasis was able to induce a marked beneficial therapeutic effect in patients compared to IMPDH inhibition.

This study was conducted according to international guidelines that were developed for the use of CsA for psoriasis [27]. Despite the existence of these guidelines, the regimens for CsA may vary substantially among countries in terms of the dose $[13,14,28]$. For CsA treatment the recommended initial dose was $2.5 \mathrm{mg} / \mathrm{kg}$ BW during the first weeks of therapy [28]. In accordance with the guidelines, the dose of CsA could be increased in the event of an insufficient response. The 12-week treatment period used was proved to be effective in previous studies with CsA or MMF, in which about $80 \%$ of the study patients had clinical improvement $[13,21]$. Previous data indicated that abrupt discontinuation of CsA medication does not increase the time to relapse compared to tapering off the CsA dose [13]. CsA is available for the treatment of moderate-to-severe psoriasis and there is ample evidence of its effectiveness from controlled and uncontrolled studies $[29,30]$. The results presented here for CsA are in agreement with those of earlier findings.

Mycophenolic acid in doses up to $7.2 \mathrm{~g}$ daily has been used several decades ago for the treatment of psoriasis, in individual patients for up to 13 years [17-19]. Most adverse events affected the gastrointestinal tract and included diarrhea, abdominal cramps and soft stools. In addition, at that time a higher incidence of viral infections, such as herpes zoster or herpes simplex, was reported [19]. The development of MMF, the ester of mycophenolic acid, was able to circumvent some of these side effects since the bioavailability was increased and lower doses were sufficient to achieve therapeutic inhibition of T cell proliferation $[31,32]$. MMF is available for the treatment of acute and chronic graft rejection in transplantation medicine. Hence, MMF was also scrutinized as an alternative for antipsoriatic therapy; however, MMF is not approved for psoriasis, and there are no guidelines for the use of MMF for psoriasis [20, 21]. In one report the change of systemic treatment from CsA to MMF to avoid CsA-induced nephrotoxicity resulted in less control of severe psoriasis in individual patients [33]. Zhou et al. [21]

demonstrated in a prospective open-label trial that MMF was able to significantly decrease the PASI score in patients. Therefore, we wanted to directly compare MMF against a classical antipsoriatic treatment such as CsA to determine the clinical efficacy of these two regimens.

Overall the tolerability of both drugs was good and no patient had to discontinue treatment because of an adverse event. Altogether, two patients had to reduce CsA medication because of hypertension. Five patients had to also reduce CsA since increased bilirubin or uric acid serum levels developed under treatment. The MMF dosage had to be reduced in 6 patients because of elevated bilirubin or liver function tests; in 1 patient MMF reduction normalized uric acid serum concentrations.

Together, the results demonstrate that CsA is more effective in controlling moderate-to-severe psoriasis whereas the tolerability and adverse events were similar in both treatment groups. Therefore, the differences between the two treatment groups with regard to efficacy, side effects, long-term use, ease of administration and costs can be used to guide treatment decisions in individual cases.

\section{Acknowledgments}

This investigator-initiated trial was supported by an unrestricted grant from Hoffmann-La Roche AG, Grenzach-Wyhlen, Germany. We would like to thank Sascha Neugebauer and Dr. Axel Hinke, WISP Wissenschaftlicher Service Pharma, Langenfeld, Germany, for help with the statistical analysis of the data. The work of WISP was supported by a grant from HoffmannLa Roche AG, Grenzach-Wyhlen, Germany.

References

Dermatology 2009;219:126-132
1 Gottlieb AB, Krueger JG, Wittkowski K, Dedrick R, Walicke PA, Garovoy M: Psoriasis as a model for T-cell-mediated disease: immunobiologic and clinical effects of treatment with multiple doses of efalizumab, an antiCD11a antibody. Arch Dermatol 2002;138: 591-600.

2 Koo J: Population-based epidemiologic study of psoriasis with emphasis on quality of life assessment. Dermatol Clin 1996;14:485496.

3 Krueger JG: The immunologic basis for the treatment of psoriasis with new biologic agents. J Am Acad Dermatol 2002;46:1-23.

4 Schön MP, Boehncke WH: Psoriasis. N Engl J Med 2005;352:1899-1912.

5 Gelfand JM, Troxel AB, Lewis JD, et al: The risk of mortality in patients with psoriasis: results from a population-based study. Arch Dermatol 2007;143:1493-1499.

6 Schön MP: Advances in psoriasis treatment. Lancet 2005;366:1367-1374. 
7 Weinstein GD, White GM: An approach to the treatment of moderate to severe psoriasis with rotational therapy. J Am Acad Dermatol 1993;28:454-459.

8 Sabat R, Philipp S, Hoflich C, et al: Immunopathogenesis of psoriasis. Exp Dermatol 2007;16:779-798.

9 Schön MP: Animal models of psoriasis: a critical appraisal. Exp Dermatol 2008;17: 703-712.

10 Berth-Jones J, Voorhees JJ: Consensus conference on cyclosporin A microemulsion for psoriasis, June 1996. Br J Dermatol 1996;135 775-777.

11 Flytström I, Stenberg B, Svensson A, Bergbrant IM: Methotrexate vs ciclosporin in psoriasis: effectiveness, quality of life and safety. A randomized controlled trial. Br J Dermatol 2008;158:116-121.

12 Heydendael VM, Spuls PI, Opmeer BC, et al: Methotrexate versus cyclosporine in moderate-to-severe chronic plaque psoriasis. $\mathrm{N}$ Engl J Med 2003;349:658-665.

13 Ho VC, Griffiths CE, Albrecht G, et al: Intermittent short courses of cyclosporin (Neoral ${ }^{\circledR}$ ) for psoriasis unresponsive to topical therapy: a 1-year multicentre, randomized study. The PISCES Study Group. Br J Dermatol 1999;141:283-291.

14 Koo J: A randomized, double-blind study comparing the efficacy, safety and optimal dose of two formulations of cyclosporin, $\mathrm{Ne}$ oral and Sandimmun, in patients with severe psoriasis. OLP302 Study Group. Br J Dermatol 1998;139:88-95.
15 Sandhu K, Kaur I, Kumar B, Saraswat A: Efficacy and safety of cyclosporine versus methotrexate in severe psoriasis: a study from north India. J Dermatol 2003;30:458463.

16 Spuls PI, Witkamp L, Bossuyt PM, Bos JD: A systematic review of five systemic treatments for severe psoriasis. Br J Dermatol 1997;137: 943-949.

17 Epinette WW, Parker CM, Jones EL, Greist MC: Mycophenolic acid for psoriasis: a review of pharmacology, long-term efficacy, and safety. J Am Acad Dermatol 1987;17: 962-971.

18 Jones EL, Epinette WW, Hackney VC, Menendez L, Frost P: Treatment of psoriasis with oral mycophenolic acid. J Invest Dermatol 1975;65:537-542.

19 Marinari R, Fleischmajer R, Schragger AH, Rosenthal AL: Mycophenolic acid in the treatment of psoriasis: long-term administration. Arch Dermatol 1977;113:930-932.

20 Haufs MG, Beissert S, Grabbe S, Schütte B, Luger TA: Psoriasis vulgaris treated successfully with mycophenolate mofetil. Br J Dermatol 1998;138:179-181.

21 Zhou Y, Rosenthal D, Dutz J, Ho V: Mycophenolate mofetil (Cellcept) for psoriasis: a two-center, prospective, open-label clinical trial. J Cutan Med Surg 2003;7:193-197.

22 Fredriksson T, Pettersson U: Severe psoriasis - oral therapy with a new retinoid. Dermatologica 1978;157:238-244.

23 Finlay AY, Kelly SE: Psoriasis - an index of disability. Clin Exp Dermatol 1987;12:8-11.

24 Finlay AY, Khan GK, Luscombe DK, Salek MS: Validation of Sickness Impact Profile and Psoriasis Disability Index in psoriasis. Br J Dermatol 1990;123:751-756.
25 Kaplan EL, Meier P: Nonparametric estimation from incomplete observations. J Am Stat Assoc 1958;53:457-481.

26 Peto R, Peto J: Asymptomatically efficient rank invariation test procedures (with discussion). J R Stat Soc A 1972;135:185-206.

27 Sterry W, Barker J, Boehncke WH, et al: Biological therapies in the systemic management of psoriasis: international consensus conference. Br J Dermatol 2004;151(suppl 69):3-17.

28 Lebwohl M, Ellis C, Gottlieb A, et al: Cyclosporine consensus conference: with emphasis on the treatment of psoriasis. J Am Acad Dermatol 1998;39:464-475.

29 Berth-Jones J, Henderson CA, Munro CS, et al: Treatment of psoriasis with intermittent short course cyclosporin (Neoral): a multicentre study. Br J Dermatol 1997;136:527530

30 Griffiths CE, Powles AV, McFadden J, Baker BS, Valdimarsson H, Fry L: Long-term cyclosporin for psoriasis. Br J Dermatol 1989; 120:253-260.

31 Behrend M: Adverse gastrointestinal effects of mycophenolate mofetil: aetiology, incidence and management. Drug Saf 2001;24: 645-663.

32 Lipsky JJ: Mycophenolate mofetil. Lancet 1996;348:1357-1359.

33 Davison SC, Morris-Jones R, Powles AV, Fry $\mathrm{L}$ : Change of treatment from cyclosporin to mycophenolate mofetil in severe psoriasis. Br J Dermatol 2000;143:405-407. 